\title{
LA NECESIDAD DE REPENSAR LA EDUCACIÓN
}

rev.ciencia.poder.aéreo.6: 17 - 23, 2011.

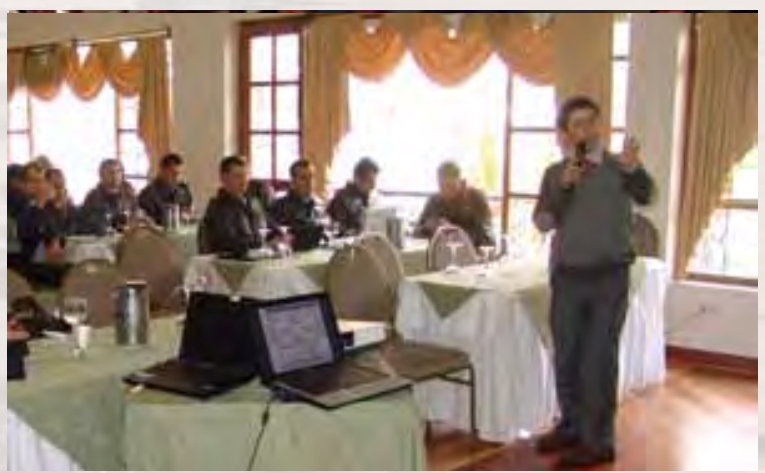

\section{Resumen}

El presente artículo tiene como objetivo generar un proceso de reflexión acerca de la educación superior y su responsabilidad en aportar a la solución de los problemas que afectan a la humanidad. Para ello, se plantea la necesidad de generar una transformación profunda en el sector educativo, en sus planteamientos, en sus modelos conceptuales, en sus prácticas, en sus concepciones del ser humano, de la realidad, del conocimiento y de la interacción, de manera que estas revoluciones conceptuales traigan consigo la implementación de novedosas prácticas, una nueva visión y un modelo educativo pertinente a las necesidades del mundo contemporáneo.
Palabras Clave: Educación, cambio, reflexión, formación, docente, conocimiento, instituciones de educación superior.

\section{Abstract}

This article aims to generate a process of reflection on higher education and its responsibility in contributing to the solution of problems affecting humanity.

To do this, there is a need to generate a profound transformation in the education sector, in their approaches, their conceptual models, in their practices, in their conceptions of human being, of reality, knowledge and interaction, so these conceptual revolutions bring with them the implementation of innovative practices, a new vision and a model of education relevant to the needs of the contemporary world.

Key Words: Education, change, reflection, training, teaching, knowledge, superior education institutions.

\footnotetext{
"Los problemas más graves que la educación debe ayudar a resolver se refieren a la lucha contra la pobreza y la exclusión y a esa falta de solidaridad que caracteriza a la sociedad... Hay que acabar con el desprecio y el
} 


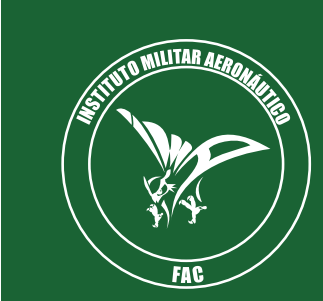

Ciencia \&

Educación

resentimiento, con la falta de respeto, con el abuso, el racismo y la separación... Con la desigualdad y la dominación. La educación debe poner las bases personales para la construcción de la justicia y la paz. Y la educación no es un fenómeno que se restringe al aula escolar. La formación ocurre también, y acaso principalmente, en el mundo de la vida."

(Constantino Carvallo Rey)

La educación tiene una responsabilidad prospectiva, la de construir el futuro.

Además de responder a problemas inmediatos y prácticos, la educación tiene el cometido de preservar las conquistas de la historia humana en los campos del conocimiento, del pensamiento, de las realizaciones artísticas y culturales, y, asimismo, anticiparse a la sociedad de su tiempo y buscar construir un futuro más acorde con los principios y valores que edifican la humanidad.

Para ello, se requiere que tanto las instituciones educativas como los educadores definan con absoluta claridad su propia concepción de sociedad, del ser humano y de sus interacciones, de la realidad y del conocimiento, pues es a partir de estas concepciones como se configuran las relaciones educativas al interior y al exterior del sistema.

Y quizás la ausencia de una postura definida y coherente en estos aspectos, ha llevado, en especial en las últimas dos décadas, a un sentimiento general de insatisfacción con la educación - en especial con el nivel educativo superior - y a la idea de solicitar reformas. Como lo reconoce la UNESCO, la educación superior ha de enfrentar imponentes desafíos y emprender la transformación y la renovación más radicales, de forma que la sociedad contemporánea, la cual en la actualidad vive una profunda crisis de valores, pueda trascender las consideraciones meramente económicas y asumir dimensiones de moralidad y espiritualidad más arraigadas (Tunnerman 2008).
En verdad, la responsabilidad de la educación no es pues, nada trivial; tal como proclama la Organización de las Naciones Unidas para la Educación, la Ciencia y la Cultura, se requiere el fortalecimiento de la pertinencia, la equidad y la internacionalización de la educación, de forma que se amplíen el acceso y la permanencia, se afiancen las relaciones de las Instituciones con las comunidades y las sociedades y se contribuya al desarrollo humano sostenible fundado en una cultura de paz, justicia social y solidaridad.

De modo específico, la UNESCO hace los siguientes encargos:

- La educación es instrumento esencial para formar ciudadanos capaces de construir una sociedad más justa y abierta.

- El conocimiento es un bien social: generado, transmitido, criticado y recreado en beneficio de la sociedad.

- La educación debe asegurar su capacidad de análisis crítico y de visión prospectiva.

- Las instituciones de Educación Superior tienen como tarea reforzar la identidad cultural de la región, participar en el mejoramiento cualitativo de todos los niveles del sistema educativo; estimular la movilidad académica y profesional; robustecer la cooperación inter-universitaria; crear y expandir los diversos tipos de redes y otros mecanismos y procesos que faciliten la integración regional y la búsqueda colectiva de equidad, calidad y pertinencia.

La adecuación del sistema educativo para que pueda responder a estas necesidades del entorno implica la generación de un proceso reflexivo y de transformación al interior de las instituciones, se necesitan nuevas concepciones de enseñanza, fundamentadas en procesos de comprensión que interpelen al educador, obligándolo a tomar posición en lo antropológico, epistemológico, ontológico y ético; de manera que tales procesos de comprensión potencien la dimensión creativa y desde ella el encuentro de sentidos y significados para su quehacer de forma pertinente. 
Según E. Díaz en Gorodokin (2005), las dificultades que se presentan en el aprendizaje a nivel del sujeto que aprende, son un reflejo de los problemas que se presentan a nivel del sujeto que enseña, transferidos de uno a otro en las diferentes etapas del proceso educativo.

Si no existe una postura clara del conocimiento, de la realidad que se estudia, del ser humano como ser que aprende y de la manera como se interactúa con los objetos, con los otros y en general, con el mundo, el docente transfiere sus creencias, prejuicios, mitos, sin una reflexión de ellos, ni de la manera en que interactúa con sus estudiantes.

De acuerdo con el mencionado autor, los obstáculos pedagógicos y epistemológicos inconscientes de los propios sujetos enseñantes, referentes a sus supuestos acerca del saber y de su propia práctica provocan resistencia al cambio o asimilación mecánica a viejos modelos, en una sociedad que cambia y que requiere otras maneras de enseñar y por ende, de aprender.

Una educación centrada exclusivamente en el profesor, en sus contenidos, rígida, disciplinar, descontextualizada de su entorno, que no promueva ideales y valores, poco contribuye a crear las condiciones para elevar los niveles de pertinencia en el conjunto del sistema.

Es necesario recuperar el papel del estudiante, de la indagación y la búsqueda, en estructuras educativas flexibles trasladando el énfasis puesto actualmente en la transmisión del conocimiento hacia el proceso de su generación, estar en contacto permanente con el contexto y el entorno y donde se promuevan e inspiren los mejores y más preciados ideales y valores de cada cultura, de forma tal que sea posible formar graduados que aprendan a aprender, a conocer, a convivir, a ser y por supuesto, a emprender.

Esto solo es posible si las mismas instituciones y los educadores inician un cuestionamiento acerca de su misión y de su quehacer en una época de tanta y tan veloz modificación.

La globalización económica y cultural y el desplazamiento hacia las economías basadas en el conocimiento, las nuevas estructuras sociales y familiares, la adecuada atención educativa, son cada vez mayores. Así mismo, existe una gran diversidad en las capacidades e intereses de los estudiantes, el fenómeno de la inmigración, la incorporación masiva de las tecnologías de la información y de la comunicación, la importancia de la capacidad de comunicación en diferentes idiomas, entre otros, que exigen otras respuestas educativas. León Trahtemberg, en su libro "La educación en la era de la tecnología y el conocimiento" (1995) realiza un amplio análisis de las exigencias de la modernidad a los sistemas educativos y a sus actores.

En cuanto al papel del educador señala lo siguiente:
Se requieren cambios paradigmáticos para girar del tradicional estilo pedagógico centrado en el profesor, la enseñanza, la información y los exámenes de ingreso a la educación superior, hacia un nuevo enfoque centrado en el estudiante, que sea capaz de pensar en asuntos que vayan más allá de las disciplinas o de sus áreas de especialización... Esto obligará a los profesores a reemplazar su actual autoimagen como recipientes de la información, por otra que los convierta en facilitadores del aprendizaje y en agentes de cambio. Deberán dejar de ser líderes transaccionales -que se apoyan en el status quo- para convertirse en líderes transformacionales, que promuevan la creación de un nuevo orden... lo que requiere el desarrollo de nuevas estrategias de enseñanza.

(Trahtemberg, 1995, p. 120).

En un mundo en rápido cambio, se percibe la necesidad de generar reflexión y transformación profunda del sector educativo, de sus planteamientos, de sus modelos conceptuales, de sus prácticas, de sus concepciones del ser humano, de la realidad, del conocimiento y de la interacción, para la consecución de ideales de sociedad, de manera que estas revolucio- 


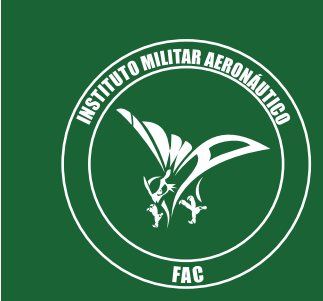

Ciencia \&

Educación

Revista Ciencia y Poder Aéreo. Edición No 6 - Septiembre 2011 ISSN 1909 - 7050

nes conceptuales traigan consigo la implementación de novedosas prácticas, una nueva visión y un nuevo modelo educativo, que debería estar centrado en el estudiante, lo cual exige reformas en profundidad, así como una renovación de los fundamentos filosóficos desde los cuales se funda la relación educativa.

Estas demandas educativas exigen la configuración de nuevas maneras de concebir el conocimiento, ya no de forma mecanicista, fragmentada, unidimensional y lineal, sino por el contrario, desde una concepción compleja, trans e interdisciplinaria; a partir del reconocimiento de la incertidumbre, de la existencia de verdades biodegradables, de la imposibilidad de lograr la completud y de la ruptura con el paradigma de la objetividad; ya no es posible concebir el conocimiento de manera simple, se requiere recurrir al pensamiento complejo para comprenderlo y hacer uso pertinente de él.

Igualmente, implica la generación de nuevas relaciones en la sociedad, donde se propenda al respeto, la equidad y la solidaridad, por un mejoramiento de las condiciones de vida de los excluidos, para que también ellos tengan acceso al conocimiento y a la información.

Esto se vuelve especialmente importante al entender que el conocimiento contemporáneo presenta un crecimiento acelerado, mayor complejidad y tendencia a una rápida obsolescencia.

Según Tünnermann, la denominada "explosión del conocimiento" es tanto cuantitativa como cualitativa, en el sentido de que se incrementa aceleradamente la cantidad de conocimiento disciplinario y, al mismo tiempo, surgen nuevas disciplinas y subdisciplinas, algunas de carácter transdisciplinario.

Según cifras de J. Appleberry, citado por José Joaquín Brunner, el conocimiento de base disciplinaria y registrado internacionalmente demoró 1.750 años en duplicarse por primera vez, contado desde el inicio de la era cristiana; luego, duplicó su volumen cada 150 y después cada 50. Ahora lo hace cada 5 años y se estima que para el año 2020 se duplicará cada 73 días (Brunner en UNESCO, 2000).

La mayor complejidad en la estructura del conocimiento contemporáneo impone una nueva manera de aproximarse a las disciplinas, ya no desde la fragmentación y de la no validación de los otros saberes, sino desde la interdisciplinariedad y la transdisciplinariedad como las maneras adecuadas de dar respuesta a esa complejidad. "La supremacía de un conocimiento fragmentado según las disciplinas, impide a menudo operar el vínculo entre las partes y las totalidades y debe dar paso a un modo de conocimiento capaz de aprehender los objetos en sus contextos, sus complejidades, sus conjuntos" (Morin, 2000, p. 18).

Como dice llya Prigogine: venimos de un pasado de certidumbres conflictivas -ya estén relacionadas con la ciencia, la ética, o los sistemas sociales- a un presente de cuestionamientos.

La incertidumbre no debe conducir a la perplejidad sino a la disposición para el cambio y a la ampliación y renovación incesante del conocimiento. Si el siglo XX fue el siglo de la búsqueda de certezas científicas y del desarrollo acelerado de las diferentes disciplinas del conocimiento humano, el presente siglo está llamado a ser el siglo de la incertidumbre y la interdisciplinariedad (Tünnermann, 2008, p. 6).

Denise Valliant afirma: "el gran desafío... es aprender a administrar la complejidad, lo que es mucho más comprometido y menos confortable que sólo pensar en ella... y que lo que han descubierto es que no hay recetas ni itinerarios fijos" (Valliant, 1999, p. 16). Ahora bien, esta demanda de un pensamiento complejo no solo se dirige a los intelectuales, es además una obligación para el sector educativo mundial.

En coherencia con este planteamiento, Miguel Ángel Escotet en Tünnermann (2008) afirma: "La gran trans- 
formación profesional que nos viene, exigirá un mayor nivel interdisciplinario, una revitalización del grupo de disciplinas relacionadas con las esferas éticas, estéticas y de comunicación, y un cambio total de actividad en profesores y estudiantes" (p.8).

De esta forma y en este contexto, se hace necesario replantear tanto en las instituciones como en el proceso de formación de los docentes, la manera como se piensa la educación y traspasar las barreras del tiempo, de manera que sea posible la autorreflexión, la autocrítica, la contextualización del saber, la multidimensionalización de la realidad, la comprensión de aquello que se quiere conocer e intervenir, y el afrontamiento estratégico de la incertidumbre.

Esto cobra especial importancia al reconocer la profesión docente como proceso que involucra a la persona en su totalidad; supone indagar, problematizar y reflexionar acerca de las concepciones, creencias, mitos, prejuicios, entre otros, así como recuperar los recorridos personales, historias escolares y modos de aprender, de pensar, de sentir y de actuar, construidos por el docente en su interacción con los contextos sociales, de manera que con todo ello pueda comprender, deconstruir y reconstruir su propio devenir, así como resignificar su práctica docente.

"Cada maestro trae una historia de vida determinada que también guía su quehacer" (Rodríguez, 2008, p. 73).

El cambio educativo se realiza de adentro hacia afuera; se requiere entonces que las instituciones repiensen su papel y su manera de concebir el conocimiento y la educación, de forma tal que modifiquen sus estructuras, sus relaciones, sus cotidianidades, sus currículos y se conviertan en instituciones flexibles, abiertas a la innovación y al aprendizaje, sólo así, se generará un entorno favorable para un cambio paradigmático en los docentes. Aún más, es indispensable otorgar el reconocimiento, la valoración, los incentivos y una excelente formación a los profesores, de manera que ellos modifiquen su rol y pasen de ser "ejecutores educativos" a "estrategas educativos".

Es por ello, que la invitación que este texto pretende hacer es que en las instituciones educativas no se exija únicamente la transformación del rol del docente, sino de manera simultánea y recurrente con él, la entidad educativa se convierta en una organización que aprende, flexible, dinámica, que reflexiona su quehacer, que mejora continuamente sus procesos, que promueve comunidades de aprendizaje y desde allí configura la creación colectiva de un nuevo rol docente más cercano a lo que requiere la sociedad del conocimiento: un docente ante todo investigador y con vocación social, reflexivo y crítico en el planteamiento y resolución de problemas pertinentes, así como en el diseño de ambientes educativos multidimensionales y de estrategias didácticas para la diversidad y la multiculturalidad.

Es necesario entonces, conjugar armónicamente concepciones epistemológicas, ontológicas, antropológicas y éticas, que le permitan mayor poder de acción al educador y que realmente aporten en la configuración de un ideal de sociedad más armónica y equitativa.

Lo que ocurre, es que generalmente en las instituciones educativas poco se reflexiona acerca de estos postulados y los educadores desarrollan su práctica docente desde sus creencias, desde su formación, en suma, desde lo que son, muchas veces cargados de prejuicios acerca de los seres humanos, lo cual a su vez, funda relaciones educativas de desconocimiento e invalidación de los otros, de transmisión de verdades que ya no responden a su nombre, de transferencia de didácticas que vivieron en su vida escolar - alejadas de aquellas requeridas desde los enfoques actuales -, con la convicción de la validez única, de la existencia de universos y la consecuente ignorancia de los multiversos, de la presencia de verdades absolutas y "ciegas"; en fin, de modelos educativos que fueron exitosos hace dos o más siglos pero que no se ajustan a las necesidades del mundo contemporáneo. 


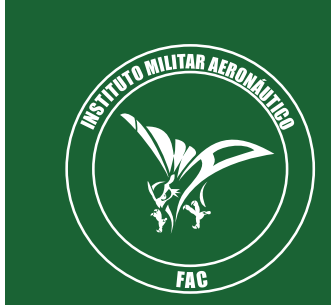

Ciencia \&

Educación

\section{Referencias}

Amartya Sen. (2005). Culture, Economics and Development. Documento preparado para la Comisión Mundial de Cultura y Desarrollo.

Bondarenko Pisemskaya, N. (2009). El componente investigativo y la formación. Docente en Venezuela en Estudios Pedagógicos XXXV, № 1: 253-260.

Universidad de Oriente, Núcleo Nueva Esparta, Isla de Margarita, Venezuela.

Berger, Paul y Luckman, Thomas (1995). La construcción social de la realidad. Barcelona: Amorrortu.Bruner, Jerome. (1991). Actos del significado. Más allá de la revolución cognitiva. Madrid: Ed. Alianza.

Brunner, J. J. (2000). Peligro y promesa: la Educación Superior en América Latina. Ensayo incluido en el libro colectivo: Educación Superior latinoamericana y organismos internacionales - Un análisis crítico (López Segrera, F. y Maldonado, A., Coordinadores) p. 93 y sigs. San Buenaventura, Cali: UNESCO, Boston College y Universidad.

Brunner y Jerome (1987). La importancia de la Educación. Paidós: España.

Carr, W. y Kemmis, S. (1988): Teoría crítica de la enseñanza. La investigación-acción en la formación del profesorado. Barcelona: Martínez Roca.

Cortina, A. (1993). Ética aplicada y democracia radical. Madrid: Tecnos.

CRESALC/UNESCO (1997). Hacia una nueva educación superior, Caracas: autor. De Zubiría, S., et al. (1998). Cultura: teorías y gestión. Pasto: Ed. Uninariño.

Delors, J. (1996): La educación o la utopía necesaria. En J. Delors (Ed.) La Educación encierra un tesoro. Informe a la UNESCO de la Comisión Internacional sobre la educación en el siglo XXI. Compendio. París: Ediciones UNESCO, pp. 9-32.

Eco, Umberto. (1975). La estructura ausente. Madrid: Ed. Lumen.

Echeverría, R. (2005). Ontología del lenguaje. Santiago: Ed. Dolmen.

Foerster, H. (1998).Por una nueva epistemología. Metapolítica. No.8.
Gimeno Sacristán, J. (1995). El currículo: ¿Los contenidos de la enseñanza o análisis de la práctica? En: Gimeno Sacristán, J. y Pérez Gómez, J. Comprender y transformar la enseñanza. Madrid: Morata. pp. 137-170.

Gómez, D. A. (2008). La Migración: el fenómeno mundial del siglo XXI. Diálogos Migrantes. Revista del Observatorio Colombo-Ecuatoriano de Migraciones OCEMI. № ${ }^{\circ} 1$. Fundación Esperanza.

González Lara, M. (2008). Paradojas en la formación docente: Elementos para avanzar en su reflexión y planteamiento de propuestas. Instituto para el Desarrollo y la Innovación Educativa IDIE - Formación de docentes y educadores. Bogotá, D.C., Colombia: Organización de Estados Iberoamericanos para la Educación, la Ciencia y la Cultura.

González Tirados R., y González, M. (2007). Diagnóstico de necesidades y estrategias de formación docente en las universidades. Universidad de La Habana, Cuba. Revista Iberoamericana de Educación. 43(6). ISSN: 1681-5653. Organización de Estados Iberoamericanos para la Educación, la Ciencia y la Cultura (OEI).

Gorodokin Ida, C. (2005, enero 1). La formación docente y su relación con la epistemología en Revista Iberoamericana de Educación, 37(5).

Guerra Zamora, P. (2007). Formación docente continua: una mirada desde el aprendizaje de adultos. Pontificia Universidad Católica de Chile.

Hunt, B. C. (2009). Efectividad del desempeño docente: una reseña de la literatura internacional y su relevancia para mejorar la educación en América Latina. Programa de Promoción de la Reforma Educativa en América Latina y el Caribe.

Imbernón, F. (2002). La investigación educativa como herramienta de formación del profesorado.

Instituto Internacional de la UNESCO para la educación superior en América Latina y el Caribe (2008). Tunnerman Bernheim, C. (s.f.) La educación superior en América Latina y el Caribe: diez años después de la conferencia mundial de 1998.

González Velasco, J. M. (2008). Reflexiones sobre la Concepción del Diseño y Desarrollo Curricular en un Mundo 
Contemporáneo y Complejo. Instituto Internacional de Integración, Convenio Andrés Bello.

Leal Soto, F. (s.f.). Efecto de la formación docente inicial en las creencias epistemológicas. Chile: Universidad de Tarapacá.

Martínez, M., Buxarrais, M. R. y Esteban, F. (2002). La Universidad como espacio de aprendizaje ético. Revista Iberoamericana de Educación, (29), pp. 17-43. Madrid: OEI.

Maturana, H. (1995). La realidad:¿Objetiva o construida? Barcelona: Ed. Anthropos.

Mayor, F. (2000). Un mundo nuevo. UNESCO.

Medina, J. (2000). Educación moral: un estudio crítico de la "clarificación de valores". Barcelona.

Morán, P. (2003). El vínculo de la docencia y la investigación México: Plaza y Valdés.

Morin, E. (1996). Introducción al pensamiento complejo. Barcelona: Ed. Gedisa, 1996.

Morin, E. (2000). Los siete saberes necesarios para la educación del futuro. Caracas: UNESCO - IESALC y Ediciones FACES / UCV. Morín de Valero, M. (2006). Investigación y formación docente. Disponible en: http://www.orestesenlared.com.ve/Colaboradores/lainvestigacion ylaformaciondocente.pdf

Omero Mora, A. (2005). Estrategia didáctica de formación docente para la enseñanza de la matemática en la escuela básica venezolana. Ciudad de La Habana.

Paniagua, M. E. (2004). La formación y la actualización de los docentes: herramientas para el cambio en educación.

Perrenoud, P. (2004). Desarrollar la práctica reflexiva en el oficio de ensenar. Barcelona: Grao.

Rodríguez Ávila, G. I. (2008). Formación de docentes para la educación en valores y ciudadanía: Tendencias y perspectivas. Instituto para el Desarrollo y la Innovación Educativa, IDIE - Formación de docentes y educadores. Bogotá, D.C., Colombia: Ediciones S.M.

Sacristán, G. y Pérez Gómez, A. I. (1992). Comprender y transformar la enseñanza. Madrid: Ed. Morata.
Searle, J. (1997). La construcción de la realidad social. Buenos Aires: Ed. Paidós.

Segura, D. (2007). El contexto de una escuela alternativa, una escuela que se hace necesaria. Ponencia, Seminario internacional sobre pensamiento latinoamericano.

Schön, D. (1988). El profesional reflexivo. Cómo piensan los profesores cuando actúan. Barcelona: Paidós.

Segal, L. (1986). Soñar la realidad. Barcelona: Ed. Paidós.

Searle, J. (1997). La construcción de la realidad social. Buenos Aires: Ed. Paidós.

Trahtemberg, L. (1995). La educación en la era de la tecnología y el conocimiento. Editorial Apoyo S. A. Torres, R. M. (1996 septiembre). Sin reforma de la formación docente no habrá reforma educativa. Perspectivas $\mathrm{N}^{\circ} 3$. París: UNESCO.

Tünnermann Bernheim, C. (2008). El rol del docente en la educación superior del siglo XXI.

UNESCO (1997). Recomendación Relativa a la Condición del Personal Docente de la Enseñanza Superior.

UNESCO (1998). Declaración Mundial para la Educación Superior en el Siglo XXI. Conferencia Mundial sobre la Educación Superior.

Vaillant, D. (2002). Formación de formadores. Estado de la práctica. Santiago de Chile: PREAL.

Von Foester, H. (1981). Notes on an epistemology of living things. Reeditado en Observing systems. Seaside, CA: Intersystems Publications. Disponible en: http://www. teamstoendpoverty.org 